\title{
TOTALLY REAL MINIMAL SUBMANIFOLDS IN A COMPLEX PROJECTIVE SPACE
}

\begin{abstract}
NORIO EJIRI
ABSTRACT. We give a pinching theorem with respect to the scalar curvatures of 4-dimensional conformally flat totally real minimal submanifolds in a 4-dimensional complex projective space.
\end{abstract}

1. Introduction. Among all submanifolds of an almost Hermitian manifold, there are two typical classes: one is the class of holomorphic submanifolds and the other is the class of totally real submanifolds. There have been many results in the theory of holomorphic submanifolds; on the other hand, there have been only a few results in the theory of totally real submanifolds.

H. Naitoh [2], M. Takeuchi [3] classified submanifolds in a real and complex space form with parallel second fundamental form.

Among such examples, there exist three $n$-dimensional conformally flat totally real minimal submanifolds in a complex projective space $P_{n}$ of constant holomorphic sectional curvature 4:

(i) a totally geodesic submanifold,

(ii) a flat torus,

(iii) a Riemannian product of $S^{1}(\sin a \cos a)$ and $S^{n-1}(\sin a)$, where $S^{n}(r)$ is an $n$-dimensional sphere with radius $r$ and $\tan a=\sqrt{n}$.

The purpose of this paper is to give a characterization of (ii) and (iii) of 4 dimension.

THEOREM. Let $M$ be a 4-dimensional compact orientable conformally flat totally real minimal submanifold in $P_{4}$. If $M$ has nonnegative Euler number and the scalar curvature $\rho$ of $M$ is between 0 and $15 / 2$, then $\rho$ is 0 or $15 / 2$ and $M$ is (ii) $(\rho=0)$, (iii) $(\rho=15 / 2)$ or its covering spaces.

Remark. If $n=4, \mathrm{~B}$. Y. Chen and K. Ogiue's result [1] implies that every compact totally real minimal submanifold in $P_{4}$ with $\rho \geqslant 64 / 7$ is totally geodesic $(\rho=12)$.

The author is grateful to Professor K. Ogiue for his useful criticism.

2. Proof of Theorem. We use the same notations and terminologies as in [1]. It was proved in [1] that the second fundamental form of the immersion satisfies

(1) $\frac{1}{2} \Delta\|\boldsymbol{\sigma}\|^{2}=\left\|\nabla^{\prime} \boldsymbol{\sigma}\right\|^{2}+\sum_{i, j} \operatorname{tr}\left(A_{i^{*}} A_{j^{*}}-A_{j^{*}} A_{i^{*}}\right)^{2}-\sum_{i, j}\left(\operatorname{tr} A_{i^{*}} A_{j^{*}}\right)^{2}+5\|\boldsymbol{\sigma}\|^{2}$.

Received by the editors January 8, 1982.

1980 Mathematics Subject Classification. Primary 53C40; Secondary 53C42.

Key words and phrases. Totally real submanifold, conformally flat, parallel second fundamental form.

(c) 1982 American Mathematical Society $0002-9939 / 82 / 0000-0389 / \$ 01.50$ 
Since

$$
\sum_{i, j} \operatorname{tr}\left(A_{i^{*}} A_{j^{*}}-A_{j^{*}} A_{i^{*}}\right)^{2}=-\sum_{i, j, k, l}\left(\sum_{m}\left(h_{k m}^{i^{*}} h_{l m}^{j^{*}}-h_{k m}^{j^{*}} h_{l m}^{i^{*}}\right)\right)^{2},
$$

this, together with the equation of Gauss, implies

$$
\sum_{i, j} \operatorname{tr}\left(A_{i^{*}} A_{j^{*}}-A_{j^{*}} A_{i^{*}}\right)^{2}=\|R\|^{2}+4 \rho-24 .
$$

By the same argument as above, we have

$$
\sum_{i, j}\left(\operatorname{tr} A_{i^{*}} A_{j^{*}}\right)^{2}=\|S\|^{2}-6 \rho+36 .
$$

Combining (1) with (2) and (3), we obtain

$$
\frac{1}{2} \Delta\|\sigma\|^{2}=\left\|\nabla^{\prime} \sigma\right\|^{2}-\|R\|^{2}-\|S\|^{2}+5 \rho .
$$

From the assumption that $M$ is conformally flat, we obtain

$$
\|R\|^{2}-2\|S\|^{2}+\frac{1}{3} \rho^{2}=0,
$$

which, together with (4), asserts

$$
\frac{1}{2} \Delta\|\sigma\|^{2}=\left\|\nabla^{\prime} \sigma\right\|^{2}-3\|S\|^{2}+\frac{1}{3} \rho^{2}+5 \rho .
$$

Taking the integrals of the both sides of it and using Green's theorem, we have

$$
\int_{M}\left\|\nabla^{\prime} \sigma\right\|^{2} * l_{M}=\int_{M}\left\{3\|S\|^{2}-\frac{1}{3} \rho^{2}-5 \rho\right\} * l_{M} .
$$

On the other hand, by the Gauss-Bonnet theorem, the Euler number $\chi(M)$ of $M$ is given by

$$
\chi(M)=\frac{1}{32 \pi^{2}} \int_{M}\left\{\|R\|^{2}-4\|S\|^{2}+\rho^{2}\right\} * l_{M} .
$$

It follows from (5) that

$$
\chi(M)=\frac{1}{32 \pi^{2}} \int_{M}\left\{\frac{2}{3} \rho^{2}-2\|S\|^{2}\right\} * l_{M} .
$$

Combining (6) with (7), we get an integral formula:

$$
48 \pi^{2} \chi(M)+\int_{M}\left\|\nabla^{\prime} \sigma\right\|^{2} * l_{M}=\int_{M} \frac{2}{3} \rho\left\{\rho-\frac{15}{2}\right\} * l_{M} .
$$

Theorem follows from the integral formula and results in $[2,3]$.

\section{REFERENCES}

1. B. Y. Chen and K. Ogiue, On totally real submanifolds, Trans. Amer. Math. Soc. 193 (1974), 257-266.

2. H. Naitoh, Totally real parallel submanifolds in $P^{n}(C)$, Tokyo J. Math. 4 (1981), 279-306.

3. M. Takeuchi, Parallel submanifolds of space forms (to appear).

Department of Mathematics, Tokyo Metropolitan University, Fukasawa, Setagaya, Tokyo, 158 JAPAN 